\title{
Nuclear Physics. The Societal Impact - A project for Greece?
}

\author{
Nicolas Alamanos ${ }^{1 *}$ \\ ${ }^{1}$ Université Paris-Saclay, IRFU, CEA, F-91191 Gif-sur-Yvette, France
}

\begin{abstract}
Answers to some of the most important questions that our world is facing out, will come from interdisciplinary efforts in medicine, energy and climate. These are involving contributions from fundamental research and in particular from nuclear physics and associated techniques. From the different types of radiation used in hospitals to Magnetic Resonance Imaging (MRI), nuclear physics and its associated technologies that is accelerators and superconducting magnets are omnipresent. The development of new radioisotope production techniques, therapy of certain cancers with ions and hadron therapy or high field MRI are among the subjects undergoing rapid development. Furthermore, archaeometry and many other societal applications are also benefited from the techniques of nuclear physics. My presentation in the 6th workshop of the Hellenic Institute of Nuclear Physics (HINP) was focused on the links between fundamental research and society and was partially inspired by an article I have published in 2020 on this subject [1]. In the last part of my presentation, I have brushed up the situation concerning the construction of low energy accelerator facilities worldwide. The construction of new accelerator installations is going through a flourishing period in particular in Europe with the construction of new accelerators dedicated to fundamental research in Nuclear Physics, to neutron production and to societal applications. This favourable climate could motivate the Hellenic nuclear physics community to design a new accelerator facility dedicated to fundamental research, neutron production and to the multiple applications of nuclear physics techniques to societal problems. Its construction could benefit from European funds and technical contributions from many European countries like France or Italy.
\end{abstract}

\section{Applications of nuclear physics techniques in medicine}

The medical imaging science started out in the beginning of last century following the discovery of Xrays by Wilhelm Röntgen. Radiography exploits the absorption of the ionizing $\mathrm{X}$ radiation when crossing the human body: the denser the tissues and the higher its average atomic number, the stronger the absorption. In radiography, the different colours or shades correspond to different levels of absorption, i.e. density and thickness.

Nearly two weeks after his discovery Wilhelm Röntgen immobilized for some moments his wife Anna Bertha's hand in front of a photographic plate in the path of the rays. After development, he observed an image of the bones in the hand. This was the first "Röntgenogram" ever taken. This first medical application of $\mathrm{X}$ rays represents one of the most obvious benefits of nuclear physics in medicine.

A century ago, the living body, like most of the material world, was opaque. Then Wilhelm Röntgen captured an X-ray image of his wife's finger - her wedding ring 'floating' around a white bone - and our vision changed forever.

\subsection{Imaging}

Positron Emission Tomography and computer Tomography (PET/CT): In order to function and reproduce cells need energy in the form of glucose. Cancer cells require a lot of energy and therefore have a very high glucose consumption. PET consists of administering intravenously to the patient a molecule marked with a radioactive isotope of a relatively short lifetime, which decays, by positron emission. An electron immediately captures the positron and the two particles annihilate each other emitting two photons each of $511 \mathrm{keV}$ [1]. The electron-positron annihilations results in gamma photons emitted at almost 180 degrees to each other; hence, it is possible, with an appropriated detection system to localize their source along a straight line. In 1969, Godfrey Hounsfield improved this technique by inventing the Computer Tomography (CT). The basic principle of a CT scanner is to take a set of X-ray images from a large number of different directions, and then to apply special algorithms to perform the so-called back-projection in order to obtain a 3D representation of the density profile of the object under observation. The large majority of PET scanners installed in hospitals are hybrid systems where a PET detector is combined with X-ray Computer Tomography (CT). According to medical specialists, the technical evolution, which led to the PET/CT technique, led to a

\footnotetext{
* Corresponding author: nicolas.alamanos@cea.fr
} 
real medical revolution. In 1979 Godfrey Hounsfield was awarded for this discovery the Nobel Prize in Physiology and Medicine.

Producing radioisotopes for medical treatment: Medical imaging is currently used for obtaining anatomical information, such as bone displacement, the condition of organs or the presence of tumours. In Europe alone there are over 12 million medical procedures per year (diagnostic and therapy) using radioisotopes, i.e. more than 30,000 procedures per day. About $90 \%$ of radioisotopes are used for diagnostic purposes and 10\% for therapy. The short-lived isotopes used in medicine require accelerators, in particular cyclotrons, nuclear reactors and possibly dedicated nuclear physics techniques for their production.

Currently, research into new and even more effective elements for medical treatment is actively being pursued in many physics laboratories. What are the reaction mechanisms for producing new radioelements? The main goal is to find efficient production routes for novel radiopharmaceuticals (theranostics, multi-modal imaging ...), with special considerations concerning purity and yield. The reaction mechanisms, the underlying theory and the most commonly used nuclear reaction codes for simulations and cross section predictions are important inputs in this field [2]. This is a good example of the application of nuclear physics techniques to other fields; codes developed for the needs of fundamental nuclear physics research are today applied to calculating the production of radioelements.

Another widely used radioisotope is ${ }^{99} \mathrm{Mo} /{ }^{99 m} \mathrm{Tc}$. Technetium- $99 \mathrm{~m}$ itself is used in more than $80 \%$ of diagnostic nuclear imaging procedures, with over 30,000 scans performed with this isotope in the United States each day and global consumption exceeding 40 million scans per year. In 2013 eight research reactors were involved in the large-scale production $(>95 \%$ of world supply) of ${ }^{99} \mathrm{Mo}$. Today three of them are shut down. Others are expected to stop irradiating targets for Technetium-99m production within the decade [3]. These supply shortages also raised interest in alternative production routes. Studies to find alternative methods for producing Technetium-99m require strong collaboration between nuclear physicists, accelerator specialists and the medical world. Several nuclear reactions have been investigated for producing Technetium-99m. Bombarding a Mo-100 target with a $22-\mathrm{MeV}$-proton beam currently seems to be one of the most effective ways for its direct production. However, the very short half-life of Technetium-99m requires that the isotope must be used quickly after production, limiting its use geographically to an area close to the cyclotron facility. Even so, for some countries, cyclotron type "methods" stand as a unique and invaluable alternative for the production of Technetium$99 \mathrm{~m}$, even if it seems necessary to pave the country with medical cyclotrons close to hospital complexes $[4,5]$. This requires innovation to provide compact, easy-touse and cost-effective cyclotrons.

When I was writing this text, I was informed that for the production of molybdenum-99 (Mo-99) other techniques, based on electron accelerators, are also under investigation [6].
Using radiations for therapy - hadron-therapy: Today, cancer is among the highest causes of death in developed countries and its treatment presents a challenge at the global level. The first to suggest the use of proton beams for radiation therapy, in 1946, was Robert Wilson, an accelerator pioneer. In conventional radiation therapy, beams of $\mathrm{X}$ rays (high-energy photons) or hadrons are produced and then projected at the patient to destroy tumour cells. Using crossing beams from many angles, radiation oncologists irradiate the tumour cells while trying to spare the surrounding normal tissues.

The strength of hadron-therapy lies in the unique physical and radiobiological properties of these particles. In particular, carbon ion beams have physical properties enabling the radiation damage to be concentrated intensively at the cancer site. These beams present a ballistic advantage with the maximum energy deposition at the end of the path (i.e. the Bragg peak) [1]. Furthermore, from the biological point of view, hadrons are more efficient in killing cancer cells and the treatment period is shorter compared to other radiotherapy methods $[7,8]$. Carbon therapy has grown up only very recently. The leading country is Japan where the first treatment centre was built in Chiba in 1994. In Germany, this type of treatment is available at the Heidelberg Ion-Beam Therapy Centre (HIT) and at the Marburg Ion-Beam Therapy Centre (MIT). In Italy, the National Centre of Oncological Hadron therapy (CNAO) also provides this treatment. Austria opened a Carbon-ion radiotherapy centre in 2017. Centres in France, South Korea, Taiwan, and China will open soon.

One of the dreams of scientists working in this field is to develop low-cost laser driven accelerators for hadron therapy. Charged particle acceleration using ultra-intense and ultra-short laser pulses has gathered strong interest in the scientific community and it is now one of the most attractive topics in relativistic laserplasma interaction research [1, 9]. Laser driven acceleration of electrons and particles may offer new possibilities for medical applications. The peculiarities of laser-driven beams led to the development of new strategies and advanced techniques for the transport, diagnostics and dosimetry of the accelerated particles, due to the wide energy spread, the angular divergence and the extremely intense pulses. In this framework, INFN-LNS (Italian Institute of Nuclear Physics, Catania) in collaboration with the ELI-Beamline Institute (Dolny Brezany, CZ) is building the ELIMED (ELI-Beamlines MEDical and multidisciplinary applications) beamline. ELIMED will be the first user addressed transport beamline dedicated to medical and multidisciplinary studies with laser-accelerated ion beams and completely open to the scientific community wishing to perform experiments with these new beams [9].

Among the open issues in this field is the compactness of the gantries [10]. Compared with protons, heavy ions are much more difficult to bend and direct to the target. Currently, HIT (Heidelberg IonBeam Therapy Centre) is the world's only carbon-ion facility with a beam that can be rotated to $360^{\circ}$. 


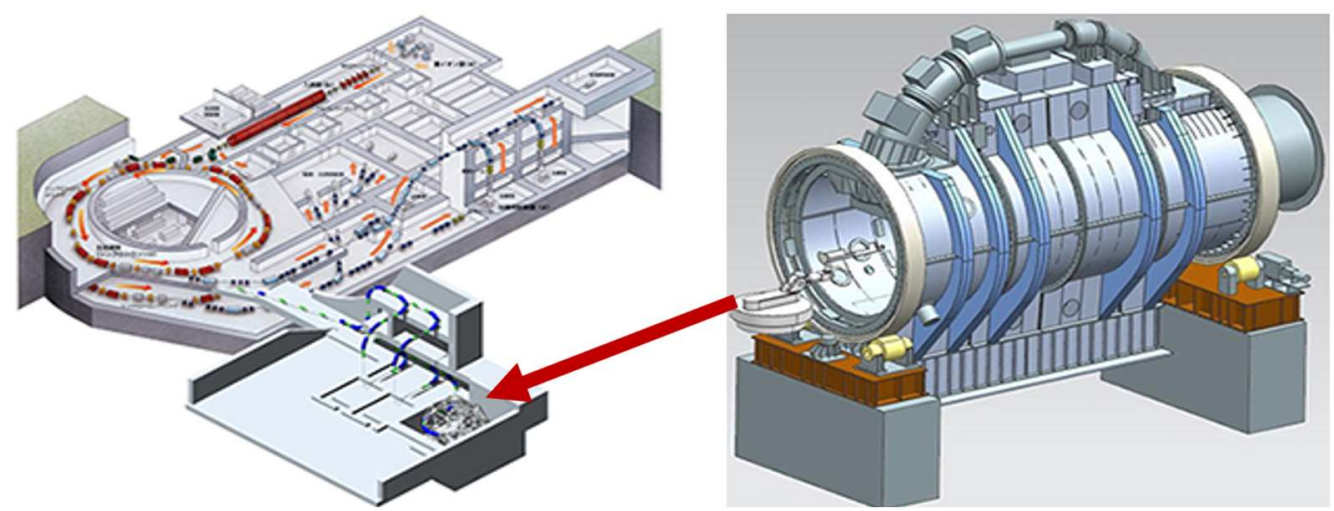

Fig. 1. Left: The Heavy-Ion Medical Accelerator in the Chiba (HIMAC) complex consists of three ion sources, one linear accelerator, two synchrotron rings, and three treatment rooms. The new treatment facility also has three treatment rooms. Right: A threedimensional image of the iso-centric rotating gantry under development for the new treatment facility.

To achieve this goal and bend the carbon beams, HIT has deployed an enormous gantry weighing 670 tons. Engineers in Nuclear and Elementary particle physics laboratories have developed techniques for building high field superconducting magnets such as the ISEULT magnet (Fig. 2). These techniques could be applied in the construction of superconducting gantries whose weight and size should be more than one-third lower than those of conventional magnets and will offer the possibility to apply higher fields.

Magnetic Resonance Imaging (MRI): To perform a study, the subject is positioned within an MRI scanner that forms a strong magnetic field around the area to be imaged. An oscillating magnetic field is temporarily applied to the patient at the appropriate resonance frequency. The only nucleus in abundance in the human body with non-zero spin is hydrogen. The excited hydrogen atoms emit a radio frequency signal, which can be measured. Hydrogen contributes two thirds to the water molecule $\left(\mathrm{H}_{2} \mathrm{O}\right)$, which forms $80 \%$ of the brain mass. MRI is a "water story" [11]. Neither oxygen nor carbon, which are key nuclei for living organisms, have spin. The larger the magnetic field, the better the image quality. For example, a field of 1.5 Tesla allows details of the brain of $\sim 2 \mathrm{~mm}$ to be imaged. A field of 11.7 Tesla would increase this resolution by a factor of 10 down to $\sim 0.2 \mathrm{~mm}$. With this resolution, high accuracy segmentation of the hippocampus becomes possible. Furthermore, highly interesting information for clinical research into Alzheimer's disease, epilepsy and schizophrenia may become available. The future of scientific research in this domain also relies on high field magnets.

In the race to increase the strength of the fields, the 11.7 Tesla ISEULT whole body magnet installed at NeuroSpin (CEA Paris-Saclay), is the magnet with the highest field existing today, see Fig. 2. It has a $900 \mathrm{~mm}$ diameter opening, capable of scanning a patient's entire body. During the tests carried out on July 18, 2019, it reached a magnetic field of 11.70 Tesla [12]. It is quite different from conventional MRI magnets. The niobium titanium superconducting wires are maintained at a very low temperature (1.8 K above absolute zero) and are powered by an excessively stable current of 1400 Amperes. The main winding of the magnet, weighing 45 tons, must be positioned as precisely as possible around the brain (a few tenths of a millimetre away).

In the coming months, many new devices will be added to the magnet to transform it into an MRI device capable of producing images of the brain. After the installation and testing of the whole system, the first images are expected to be produced in the end of the year.

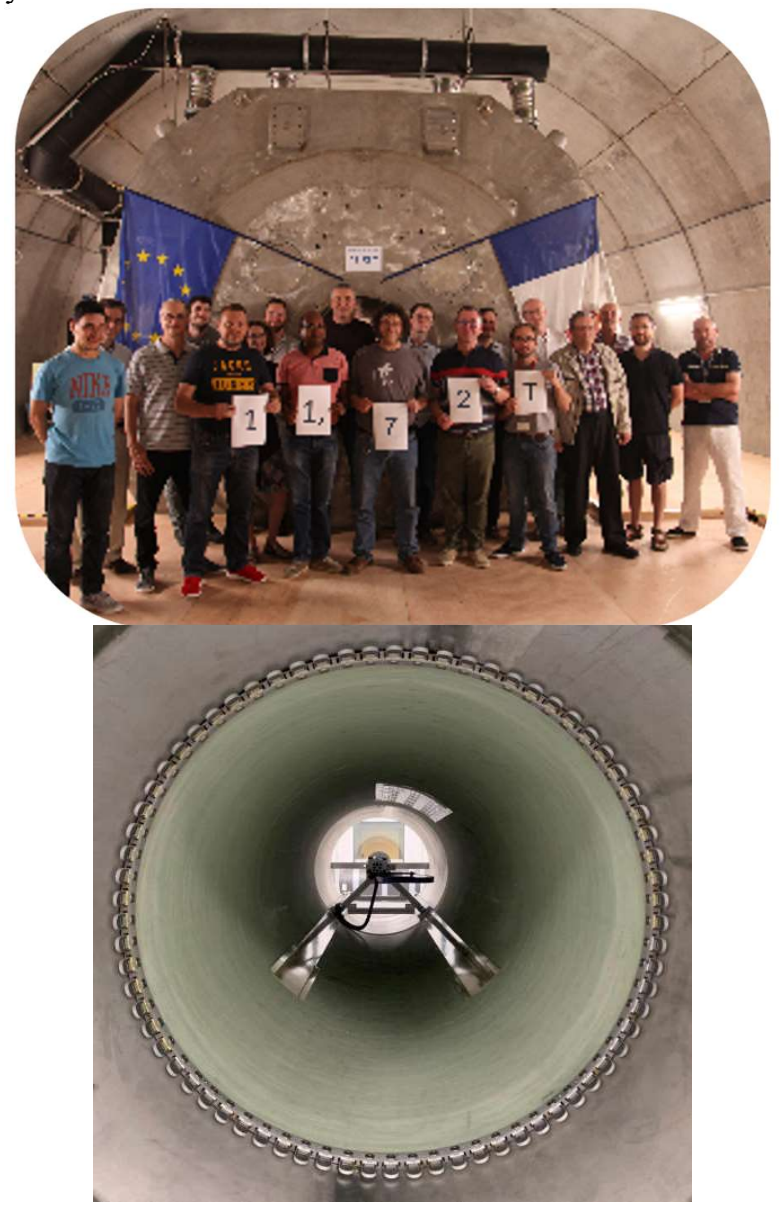

Fig. 2. Top: With the new record of 11.7 Tesla, ISEULT exceeds the most powerful MRI magnet currently in service in the world, based in Minneapolis, reaching 10.5 Tesla, but with a smaller central opening of $880 \mathrm{~mm}$, compared to $900 \mathrm{~mm}$ for ISEULT. Bottom: The magnetic field must be homogeneous at 0.5 PPM (parts per million) around the patient's brain. 5904 pieces of shim (small iron pellets) were thus screwed onto their rails and installed inside the magnet tunnel. 


\section{Other societal applications}

If the medical field is the one where the contributions of nuclear physics are probably the most remarkable, it is not the only beneficiary. Archaeometry and in particular dating, air pollution measurements, and "neutron" techniques for safety investigations are among the beneficiaries of the instrumentation developed in nuclear physics laboratories.

Cultural heritage - Archaeometry: The purpose of archaeology is to examine, interpret and date archaeological remains. Many nuclear physics methods are used to analyse the composition of archeometrical samples. Among these, the most popular are probably the PIXE and PIGE (Particle Induced X-ray Emission) and (Particle Induced $\gamma$-ray Emission) methods, providing information on the "elementary" composition (in the sense of chemical element) of a sample (Z), and the RBS (Rutherford Back Scattering) method providing information on the thickness and composition (A) of the sample. These techniques allow the state of preservation of artworks and finds to be studied, the support of restoration (to find for instance the most appropriate materials which are compatible with the original ones), and the study of artistic techniques and the dating of samples [1, 13].

Dating, and in particular the ${ }^{14} \mathrm{C}$ method, is probably among the greatest contributions of Nuclear Physics to Archaeology. How does it work? Cosmic rays collide with atoms in the atmosphere creating energetic neutrons. These neutrons collide with nitrogen atoms creating ${ }^{14} \mathrm{C}$ via the $\left(\mathrm{n}+{ }^{14} \mathrm{~N} \rightarrow \mathrm{p}+{ }^{14} \mathrm{C}\right)$ reaction. ${ }^{14} \mathrm{C}$ is radioactive, decaying by $\beta$ emission, with a half-life of about 5,700 years. The ${ }^{14} \mathrm{C}$ atoms combine with oxygen to form carbon dioxide $\left(\mathrm{CO}_{2}\right)$, which is absorbed and incorporated into plant fibres by photosynthesis. Animals and people eating the plants absorb ${ }^{14} \mathrm{C}$ too. The ratio of carbon ${ }^{12} \mathrm{C}$ to ${ }^{14} \mathrm{C}$ in the air and in all living organisms is nearly constant at any time.

The ${ }^{14} \mathrm{C}$ atoms are always decaying, but new ${ }^{14} \mathrm{C}$ atoms are replacing them at a constant rate. Our bodies have a certain percentage of ${ }^{14} \mathrm{C}$ atoms in them, and all living plants and animals have the same percentage. When an organism dies, carbon exchange with the biosphere ceases, it stops absorbing ${ }^{14} \mathrm{C}$ and the ${ }^{14} \mathrm{C}$ isotope content decreases at a rate set by the laws of radioactive decay. For this reason, the ratio between ${ }^{12} \mathrm{C}$ and ${ }^{14} \mathrm{C}$ gives precise information on the date of death.

Dating is based on a comparison of the ratio of ${ }^{12} \mathrm{C}$ and ${ }^{14} \mathrm{C}$ contained in a sample with that of a standard reference sample. From this comparison, the ${ }^{114} \mathrm{C}$ age" of the sample is deduced, Fig. 3. This ${ }^{14} \mathrm{C}$ age" is then translated into real age (or "calendar age"). There is a possibility that the ${ }^{14} \mathrm{C} /{ }^{12} \mathrm{C}$ ratio might have varied over time and that a correction will need to be applied to radiocarbon ages to obtain calendar dates [13]. The most accurate method for obtaining the ${ }^{14} \mathrm{C} /{ }^{12} \mathrm{C}$ ratio is accelerator mass spectroscopy (AMS). The ions are accelerated before mass analysis [13]. Their special strength relies on the power to separate a rare isotope from an abundant neighbouring mass ("abundance sensitivity", e.g. ${ }^{14} \mathrm{C}$ from ${ }^{12} \mathrm{C}$ ). AMS makes possible the detection of naturally occurring, long-lived radioisotopes such as ${ }^{10} \mathrm{Be},{ }^{36} \mathrm{Cl},{ }^{26} \mathrm{Al}$ and ${ }^{14} \mathrm{C}$ with typical isotopic abundancies ranging from $10^{-12}$ to $10^{-18}$.

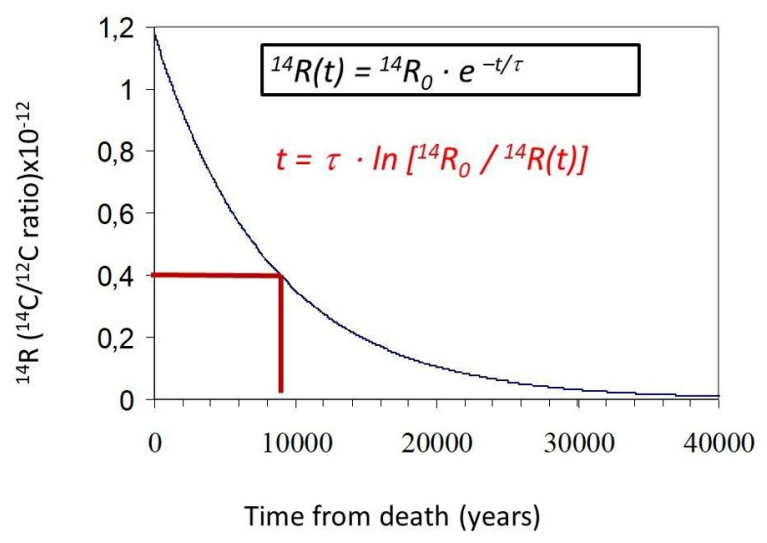

Fig. 3. ${ }^{14} \mathrm{C}$ is used as a natural chronometer to date organic materials, those systems that have been directly (or indirectly) in equilibrium with the atmosphere, acquiring ${ }^{14} \mathrm{C}$ from it, and that have ceased that equilibrium condition at a well-defined moment.

A few years ago, the ${ }^{14} \mathrm{C}$ dating method was brought to public prominence thanks to the dating of the shroud of Turin. The shroud of Turin is a linen cloth that tradition associates with the crucifixion and burial of Jesus. It has undergone numerous scientific tests, the most notable of which is radioactive dating. In 1988, scientists at three separate laboratories dated samples from the Shroud to a range between 1260 and 1390 AD, which coincides with the first certain appearance of the shroud in the 1350 s much later than the burial of Jesus in 30 or $33 \mathrm{AD}$.

The ${ }^{14} \mathrm{C}$ dating method is not just applied to old (or very old!) objects. Indeed, nuclear test explosions performed after the Second World War injected a large amount of neutrons into the atmosphere that induced a huge increase in atmospheric ${ }^{14} \mathrm{C}$ concentration. The effect was so considerable that after 1955, in less than ten years radiocarbon concentration almost doubled, reaching its maximum value in 1963-1965 [13]. This effect helped to date a supposed Fernand Léger painting (Contraste de Formes) allegedly painted in 1913-14. It was bought by Peggy Guggenheim in the late 1960s but never displayed to the public because of early suspicions that it was a forgery. From the data shown in Fig. 4 it appears obvious that the painting is a fake $[14,15]$. This method is also used to date great Bordeaux wines.

The accuracy and precision of radiocarbon $\left({ }^{14} \mathrm{C}\right)$ dating depend on several factors affecting the main steps of the dating process, from the selection of the samples to their preparation and measurement, involving a systematic evaluation of anomalies potentially introduced during each stage of the process and attempting to minimize the uncertainties. Archeometrical methods are at the crossroads of several scientific fields. Supported by sophisticated technologies, they refine our vision of world Cultural Heritage. This results in renewed interest in analytical pipelines, data management and preservation plans for cultural remains. 

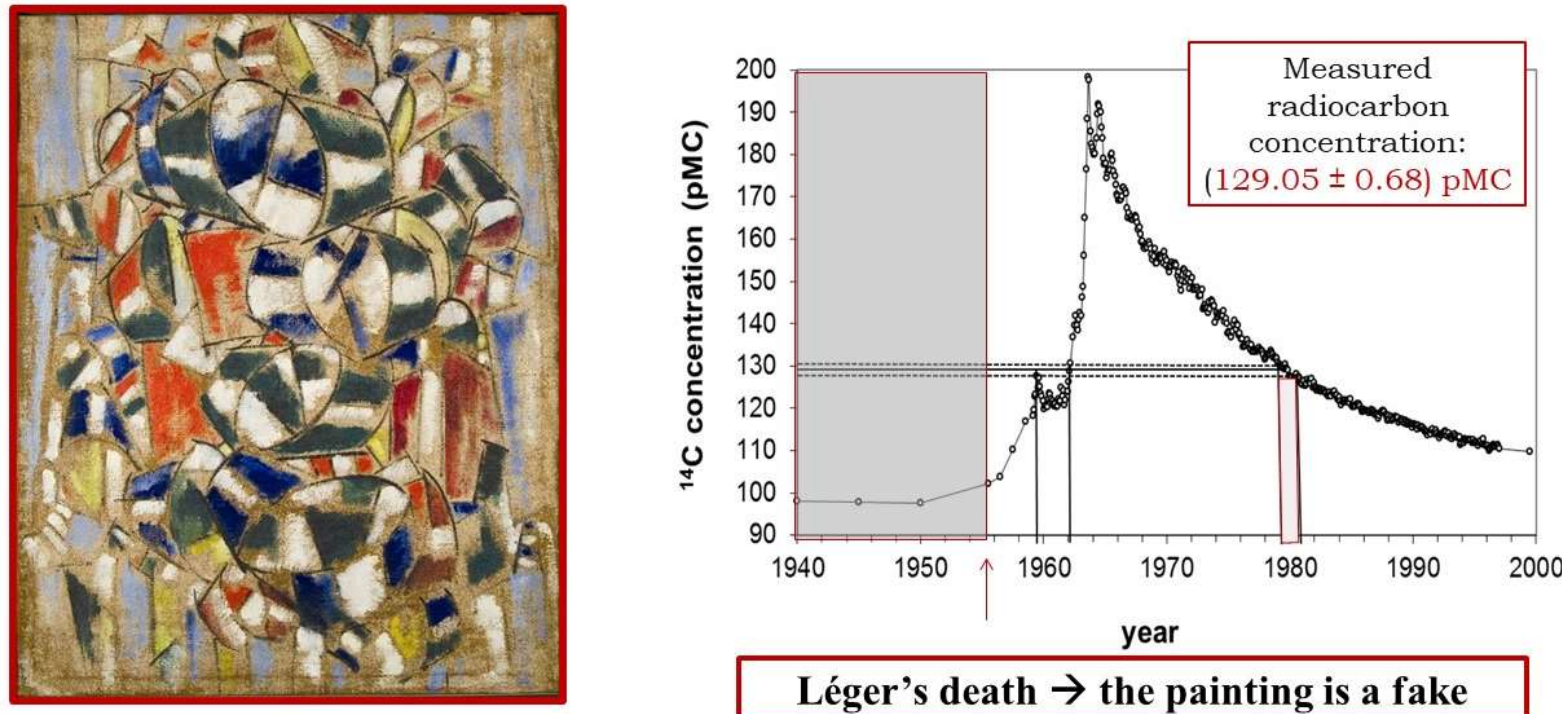

Fig. 4. The black curve represents the ${ }^{14} \mathrm{C}$ concentration increase until 1963-1965 due to nuclear test explosions. From the ${ }^{14} \mathrm{C}$ concentration, it appears obvious that the canvas was produced using cotton plants harvested in 1959, 1962, or 1979-80, I.e. after Fernand Léger's death on August 17, 1955 in Gif-sur-Yvette.

\section{The worldwide landscape concerning emerging accelerator facilities}

Several accelerator facilities are under construction in the world for satisfying the needs of fundamental research, neutrons production or demands associated to societal applications.

Fundamental research: The international scientific landscape concerning the production of radioactive beams has been profoundly enriched with the commissioning of new facilities such as FAIR (Facility for Antiproton and Ion Research) in Germany, FRIB (Facility for Rare Isotope Beams) at Michigan State University or ARIEL (Advanced Rare Isotope Laboratory) at TRIUMF. FAIR is aiming to accelerate a large variety of particle beams of a previously unparalleled intensity and quality, as well as antiprotons. FRIB is a truly next generation projectilefragmentation facility with a $200 \mathrm{MeVA} 400 \mathrm{~kW}$ LINAG followed by a fragmentation separator. Few months ago, April $16^{\text {th }}$, FRIB reached an important milestone with first acceleration of beam in ReA6. A ${ }^{14} \mathrm{~N}^{6+}$ beam was accelerated to $10.2 \mathrm{MeV} / \mathrm{u}$ using the new ReA6 beta $=0.085$ cryomodule with all cavities at full field. ARIEL at TRIUMF with the use of a super-conducting electron LINAG, a new $30 \mathrm{MeV}$ electron linear accelerator, is aiming to obtain $\sim 10^{14}$ fission/s. Other countries have decided to build their own radioactive beam facilities such as RISP (Radioactive Isotope Science Project) in KOREA, Beijing ISOL (Isotope Separation On Line) in CHINA or DERICA (Dubna Electron-Radioactive Ion Collider fAcility) at DUBNA in RUSSIA. A new project is also under consideration at GANIL France. Furthermore, intermediate scale facilities as the HIE ISOLDE at CERN a natural extension of the present facility by the addition of a $10 \mathrm{MeVA}$ post-accelerator has been constructed or SPES at INFN-Legnaro a national $8 \mathrm{~kW}-\mathrm{ISOL}$ is under construction.

Compact accelerators for neutron production: Since several decades, nuclear reactor based neutrons sources, have proven their benefit in supporting science, industry, agriculture and health. However, the neutrons landscape worldwide and in particular in Europe will change profoundly over the next few decades [16]. The ORPHEE reactor in France was recently shut down and others like the BER2 in Germany, REZ in the Czech Republic, BRR in Hungary or Democritus in Greece and eventually ILL in Grenoble are expected to be shut down in the very near future. Therefore, two-thirds of currently operating neutron sources in Europe will close within the next few decades. Even if an increase in the capacity of spallation sources will take place with the increase of ISIS capacities and the commissioning of ESS, this situation is excessively penalizing for the 6000 users of neutrons in Europe. One hope for coping with this worldwide shortage of neutron sources comes from a possible massive deployment of Compact Accelerator Neutron Sources (CANS) [17]. What is it all about? A CANS is accelerating a very high intensity beam of protons or deuterons, typically from 5 to $100 \mathrm{~mA}$, at energies between a few $\mathrm{MeV}$, typically from $3 \mathrm{MeV}$ to $5 \mathrm{MeV}$ up to $\sim 40 \mathrm{MeV}$. After acceleration, the beam impinges on a dedicated neutron production target, which may be surrounded by a moderator for providing epithermal and thermal neutron beams. Low energy CANS, using deuteron and proton beams of energy between $3 \mathrm{MeV}$ and $5 \mathrm{MeV}$ are attractive because they are relatively inexpensive, $(\sim 10 \mathrm{M} €)$ and can be installed and operated relatively easily, for instance in University environments. This type of CANS can be used for a variety of applications such as radiography, radioelements production for nuclear medicine; etc. but also for educational purposes and less demanding in terms of neutron flux experiments (see the JCANS network in Japan [18]). Their construction uses 
techniques perfectly mastered nowadays, i.e. a proton or deuteron source of $\sim 5 \mathrm{~mA}$ up to $100 \mathrm{~mA}$ and a radiofrequency quadrupole (RFQ) to reach energies between $3 \mathrm{MeV}$ and $5 \mathrm{MeV}$. Nevertheless, the blocking point for this type of development is the neutron production target. For proton energies below $3 \mathrm{MeV}$, lithium should be preferred due to the low stripping reaction threshold and a higher neutron production cross section. For proton energies higher than $3 \mathrm{MeV}$, beryllium and lithium are roughly equivalent in terms of neutron yield [19].

Demands associated to societal applications: Supporting the knowledge of artworks past times and civilizations by nuclear physics technics is a very valuable intellectual contribution to the historical and archaeological studies. Every important museum, like the Acropolis museum, has to dispose or to be associated with an accelerator facility and eventually participate in collaboration with Nuclear Physics Laboratories to the construction of experimental setups aiming to perform in situ measurements. This is for instance the goal of MACHINA, the first transportable proton accelerator facility based on RFQ cavities, jointly developed by INFN and CERN and specifically designed to perform in situ cultural heritage studies [13].

\section{Outlook}

Recent developments in accelerator technologies have led several countries to consider the possibility of building medium-size facilities with the objective of promoting for large number of disciplines national research. An example of this type of initiatives is the SARAF project in Israel. SARAF will be a user facility for basic and applied nuclear physics. The research plans for this facility span several disciplines: Precision studies beyond the Standard-Model. Extended nuclear astrophysics research with high-energy neutrons. Studies of exotic neutron-rich isotopes relevant to the rprocess. Nuclear structure of exotic isotopes. Highenergy neutron cross section measurements for basic nuclear physics and material science research. Neutron based imaging, therapy, novel radiopharmaceuticals development, and production [20].

A SARAF type facility, eventually financed by European funds, could be also consider for Greece. I am convinced that many European countries will be eager to participle in its contraction. I would like to remind that the prize of such a facility is a fraction, between 25 and $50 \%$, of the prize of a football player like the French player Kylian Mbappé. Into this very favourable context, the Hellenic nuclear physics community could contribute by nominating a scientific and technical group aiming to design and propose a national project. This project, if accepted by the national authorities, should boost not only nuclear physics but also the ensemble of the scientific fields using its techniques. It would also help to create a multicultural centre and increase the number of young scientists in Greece.

\section{References}

1. Nicolas Alamanos, "An introduction to the school "Rewriting Nuclear Physics Textbooks: one-step forward" and future perspectives", Eur. Phys. J. Plus 135, 417 (2020).

2. A. Fontana and L. Canton, "Nuclear Physics applied to the production of Innovative RadioPharmaceuticals", Eur. Phys. J. Plus (to be published).

3. R. Van Noorden, The medical testing crisis, Nature 504, 202.

4. P. Schaffer et al., "Direct Production of $99 \mathrm{mTc}$ via ${ }^{100} \mathrm{Mo}(\mathrm{p}, 2 \mathrm{n})$ on Small Medical Cyclotrons", 23rd International Conference on the Application of Accelerators in Research and Industry (CAARI).

5. J. Tanguay et al., "A fast and simple dosecalibrator-based quality control test for the radionuclide purity of cyclotron-produced 99mTc", Phys. Med. Biol. 60, 8229-8247 (2015) and "Cyclotron based production of $99 \mathrm{mTc}$ ", IAEA report.

6. Press Releases, May 11th, 2021, https://www.northstarnm.com/northstar-medicalradioisotopes-receives-electron-beam-acceleratorsfor-first-of-its-kind-advanced-medicalradioisotope-production/

7. H. Tsujii, Overview of Carbon-ion Radiotherapy, IOP Conf. Series: Journal of Physics: Conf. Series 777, 012032 (2017).

8. D. Kramer, Carbon-ion cancer therapy shows promise, Physics Today 68, 6, 24 (2015).

9. D. Margarone, G.A.P. Cirrone et al., "ELIMAIA: A Laser-Driven Ion Accelerator for Multidisciplinary Applications", Quantum Beam Sci. 2, 8 (2018).

10. Y. Iwata et al., Superconducting gantry and other developments at HIMAC, Proceedings of PAC2013, Pasadena, CA USA.

11. D. Le Bihan, « Le cerveau de cristal : Ce que nous révèle la neuro-imagerie ». Odile Jacob. Paris, 2012.

12. L. Quettier et al., IEEE Transactions on applied superconductivity, Vol. 28, No. 3, April 2018.

13. M. Fedi, "How a small accelerator can be useful for interdisciplinary applications part II: cultural heritage studies", Eur. Phys. J. Plus 136, 411 (2021)

14. L. Caforio, et al, Eur. Phys. J. Plus 129, 6 (2014)

15. M. Fedi et al, Nuclear Instruments and Meth. B 294, 662 (2013).

16. ESFRI Physical Sciences and Engineering Strategy Working Group Neutron Landscape Group. ISBN: 978-88-901562-5-0

17. I.S. Anderson, C. Andreani, J.M. Carpenter, G. Festa, G. Gorini, C.-K. Loong, and R. Senesi, « Research opportunities with compact acceleratordriven neutron sources»), Physics Reports 654, 158 (2016). 
18. http://www.jcans.net/

19. O. Sgouros, V. Soukeras and A. Pakou, "Low energy proton induced reactions with weakly bound nuclei for application purposes", Eur. Phys.

J. A, 57, 125 (2021).

20. I. Mardor et al., Eur. Phys. J. A 54, 91 (2018) 